\title{
Nuclear-Pumped Lasers for Large-Scale Applications
}

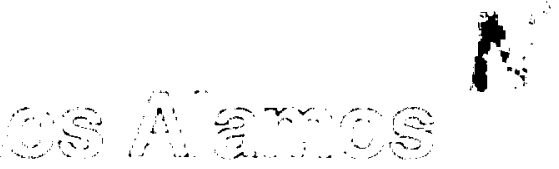

Los Alamos Nafumal Latherafory is operafed by the Unitersity of Califome tor the Unted States Department of Enerye under contract W.7415-ENG.30. 
Edited by Gerry Edwards, Group IS-11

Composition and illustrations by Gary W. Webb, Group N-2

Manuscript prepared by Dollie Lucero, Group N-2

An Affirmatrive Action/Equal Opportunity Employer

This neport was prepured as an account of work sponsoned by an agereycy of the Unitad Sutes Govermment. Neither the United Stotes Government nor any acency thereof.

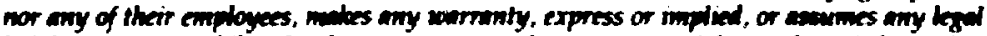
linbility or responsibility for the accuracy. completeness, or usefulmess of any informetion.

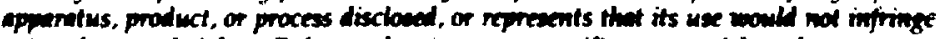
privusely owned rights. Reference herein to any specific commenciel product, process, or

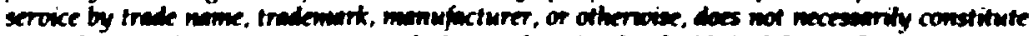

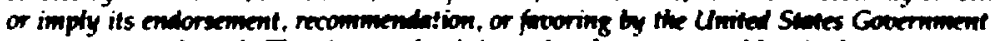
or any agency thereof. The views and opinions of wuthors expmesucl herein do not mecessarity state of nefect those of the United Sintes Goorrmment or any agency thereof. 
Nuclear-Pumped Lasers

for Large-Scale Applications
R. E. Anderson
E. M. Leonard
R. F. Shea
R. R. Berggren

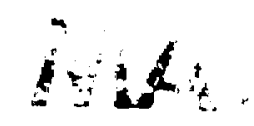




\title{
NUCLEAR-PUMPED LASERS FOR LARGE-SCALE APPLICATIONS
}

\author{
by
}

\author{
R.E. Anderson, E.M. Leonard, R.F. Shea, and R.R. Berggren
}

\begin{abstract}
Efficient initiation of large-volume chemical lasers may be achieved by neutron induced reactions which produce charged particles in the final state. When a burst mode nuclear reactor is used as the neutron source, both a sufficiently intense neutron flux and a sufficiently short initiation pulse may be possible. Proof-of-principle experiments are planned to demonstrate lasing in a direct nuclear-pumped large-volume system; to study the effects of various neutron abeorbing materials on laser performance; to study the effects of long initiation pulse lengths; to demonstrate the performance of large-scale optics and the beam quality that may be obtained; and to assess the performance of alternative designs of burst systems that increase the neutron output and burst repetition rate.
\end{abstract}

\section{INTRODUCTION}

There has been considerable interest, both in the past ${ }^{1}$ and more recently ${ }^{2,3}$ in the issue of nuclear pumping of gas laser media. Two characteristics of a nuclear reactor that have fueled this interest are the high-energy output that is coupled with a relatively small, self-contained system. These features provide mechanisms for overcoming the restrictions imposed by volume, weight, or maintenance-limited systems. The long mean-free paths associated with uncharged particles such as the neutron also provide a convenient mechanism for pumping large-volume gas systems.

We have been interested in the possibility of using neutrons to initiate a $\mathrm{DF}-\mathrm{CO}_{2}$ transfer laver. This type of chemical laser has an inherent advantage over excimer lasers, because the output energy arises as a result of chemical reactions among the constituents of the laser medium rather than as a result of the energy supplied by an external pumping device. In the DF- $\mathrm{CO}_{2}$ laser, the neutron pump only provides sufficient energy to initiate the chemical reaction chain. The laser may then produce an output energy approximately ten times larger than that supplied by the pump, compared to a conventional system which might produce an output energy equal to a few percent of the pump energy.

In the reactor pumping concept, neutrons produced by the reactor are absorbed by a nucleus within the lasing medium. The nucleus then undergoes a reaction that produces highly energetic charged particles, which deposit energy in the laser gas. This is similar to the process in an electron-beam-pumped laser. 
To accomplish this process most efficiently, the nuclei that dope the laser must have a large absorption cross section for low-energy neutrons, a large positive q-value for the nuclear reaction, and must not substantially perturb the operation of the laser. It would also be advantageous if the species of interest were to exist in a gaseous form. Several candidates that have received considerable attention in the literature ${ }^{1-3}$ are ${ }^{3} \mathrm{He},{ }^{6} \mathrm{Li},{ }^{10} \mathrm{~B}$, and ${ }^{235} \mathrm{U}$. The relevant properties of these isotopes are listed in Table I. The quantities of these nuclei that must be preeent vary considerably, depending on the energy release per absorption event, as discussed in Sec. V. For example, uranium hexafluoride needs to be present only in very small quantities. The addition of any of these species raises complex questions regarding its effect on the laser kinetics. For some species (such as ${ }^{3} \mathrm{He}$ ), we have enough information to predict DF-CO $\mathrm{CO}_{2}$ laser operation if a quantity of ${ }^{3} \mathrm{He}$ were added to the gas mixture.

\begin{tabular}{|c|ccc|}
\hline & \multicolumn{3}{|c|}{ TABLE I. PROPERTIES OF LASER ADDITIVES } \\
\hline Isotope & Thermal Cross Section & Energy Release & Availability \\
\hline${ }^{3} \mathrm{He}$ & $5300 \mathrm{~b}$ & $0.76 \mathrm{MeV}$ & gas \\
\hline${ }^{6} \mathrm{Li}$ & $950 \mathrm{~b}$ & $4.8 \mathrm{MeV}$ & \\
\hline${ }^{10} \mathrm{~B}$ & $3800 \mathrm{~b}$ & $2.3 \mathrm{MeV}$ & gas \\
\hline${ }^{235} \mathrm{U}$ & $580 \mathrm{~b}$ & $170.0 \mathrm{MeV}$ & gas \\
\hline
\end{tabular}

\section{BFFECT OP HEIUM ON LASER KINETICS}

Detailed studies have been performed on photolytically initiated and, more recently, on e-beam initiated DF-CO2 transfer lasers. Recent unpublished experiments on these systems have shown that weakly initiated systems can provide reasonable energy extraction $(-30 \mathrm{~J} / l)$ with lasing pulses (on-time to off-time) longer than $50 \mu \mathrm{s}^{4}$ We will use the parameter space investigated by these experiments as a point of departure. Therefore we will fix the total operating pressure at $1 \mathrm{~atm}$ and the gas composition as shown in Table II. For neutron initiation we will replace $10 \%-50 \%$ of the argon bufker gas with ${ }^{3} \mathrm{He}$.

\begin{tabular}{|c|c|}
\hline \multicolumn{2}{|c|}{ TABLE II. GAS COMPOSITION FOR DF-CO2 LASER } \\
\hline Components & Pressure (torr) \\
\hline Buffer (argon) & 664 \\
\hline $\mathrm{F}_{2}$ & 45 \\
\hline $\mathrm{D}_{2}$ & 15 \\
\hline $\mathrm{CO}_{2}$ & 35 \\
\hline $\mathrm{O}_{2}$ & 1 \\
\hline Total pressure & 760 \\
\hline
\end{tabular}


The basic kinetics of the system are straightforward. The laser is initiated by producing free F-atoms that set off the $D_{2}, F_{2}$ chain:

$$
\begin{aligned}
& F+\mathrm{D}_{2} \rightarrow \mathrm{DF}(\mathrm{v})+\mathrm{D} \\
& \mathrm{D}+\mathrm{F}_{2} \rightarrow \mathrm{DF}(\mathrm{v})+\mathrm{F}
\end{aligned}
$$

The vibrational energy in DF may then be transferred to $\mathrm{CO}_{2}$,

$$
\mathrm{DF}(\mathrm{v})+\mathrm{CO}_{2}(000) \rightarrow \mathrm{DF}(\mathrm{v}-1)+\mathrm{CO}_{2}(001) \quad,
$$

which lases at $10.6 \mu$ :

$$
\mathrm{CO}_{2}(001) \rightarrow \mathrm{CO}_{2}(100)+\mathrm{hv}(10.6 \mu)
$$

The production of free $\mathrm{F}$-atoms in these devices is most conveniently provided by photolysis of $F_{2}$ :

$$
\mathrm{F}_{2}+\mathrm{hv}(280 \mu) \rightarrow \mathrm{F}+\mathrm{F}
$$

An e-beam may also be used to provide free $F$-atoms in the $A r, F_{2}$ mixture. Since neutron abeorption and subsequent fission of ${ }^{3} \mathrm{He}$ produces energetic charged particles, the neutron-initiated system acts very much like e-beam initiation.

The system is clearly unstable: any inadvertent production of F-atoms (such as that due to fluorescent lamps) could cause the laser to fire. Pre-ignition is prevented by the addition of a "chain breaker" that acts to remove free $F$ and $D$ atoms. The chain breaker also sets the threshold, which the pump must exceed to fire the laser. The laboratory experiments mentioned above have used 1-2 torr of $\mathrm{O}_{2}$ for this purpose.

In the transfer system there are only a few reactions where the buffer gas plays a major role. These processes are listed below.

$$
\begin{aligned}
& \mathrm{CO}_{2}(001)+\mathrm{M} \rightarrow \mathrm{CO}_{2}(030)+\mathrm{M} \\
& \mathrm{CO}_{2}(010)+\mathrm{M} \rightarrow \mathrm{CO}_{2}(000)+\mathrm{M} \\
& \mathrm{DF}(\mathrm{v})+\mathrm{M} \rightarrow \mathrm{DF}(\mathrm{v}-1)+\mathrm{M} \\
& \mathrm{D}+\mathrm{O}_{2}+\mathrm{M} \rightarrow \mathrm{DO}_{2}+\mathrm{M} \\
& \mathrm{F}+\mathrm{O}_{2}+\mathrm{M} \rightarrow \mathrm{FO}_{2}+\mathrm{M}
\end{aligned}
$$

In Table III we give rate constants for the first three of these processes at representative temperatures and with $\mathrm{M}$ as either argon or helium. We have used the $\mathrm{CO}_{2}$ rate constants recommended by Feber and Sullivan ${ }^{5}$ and the DF rates recommended by Cohen and Bott. ${ }^{6}$

Reaction 4 has the same rate ${ }^{7}$ with either $M=A r$ or $M=$ He. No data exist on Reaction 5 with helium as a third body, and we have assumed the same rate for both helium and argon.

Because of the rapid deactivation rate of the $\mathrm{CO}_{2}$ bending mode by helium (Reaction 2), most $\mathrm{CO}_{2}$ lasers use it as a buffer gas. All three reports of e-beam-initiated $\mathrm{DF}-\mathrm{CO}_{2}$ lasers in the literature used helium. $^{8-10}$ Our system, however, operates in a different regime, where deactivation of the bending mode is less critical. Performance limitations on the long pulse DF- $\mathrm{CO}_{2}$ laser are more dependent upon quenching of the upper laser level, whtre argon and helium have comparable rates. 


\begin{tabular}{|c|c|c|c|c|}
\hline \multicolumn{3}{|c|}{ TABLE III. COMPARISON OF KINETIC RATES INVOLVING ARGON AND HELIUM } \\
\hline Reaction & $\mathrm{M}$ & $300 \mathrm{~K}$ & $600 \mathrm{~K}$ & $800 \mathrm{~K}$ \\
\hline $\mathrm{CO}_{2}(001)+\mathrm{M}$ & $\mathrm{Ar}$ & $1.56 \times 10^{-15}$ & $1.72 \times 10^{-14}$ & $5.11 \times 10^{-14}$ \\
\cline { 2 - 5 } & $\mathrm{He}$ & $2.30 \times 10^{-15}$ & $1.86 \times 10^{-14}$ & $5.69 \times 10^{-14}$ \\
\hline $\mathrm{CO}_{2}(010)+\mathrm{M}$ & $\mathrm{Ar}$ & $9.33 \times 10^{-16}$ & $1.63 \times 10^{-14}$ & $4.46 \times 10^{-14}$ \\
\cline { 2 - 6 } & $\mathrm{He}$ & $1.17 \times 10^{-13}$ & $8.29 \times 10^{-13}$ & $1.65 \times 10^{-12}$ \\
\hline \multirow{2}{*}{$\mathrm{DF}(1)+\mathrm{M}$} & $\mathrm{Ar}$ & $3.73 \times 10^{-18}$ & $8.43 \times 10^{-17}$ & $3.08 \times 10^{-16}$ \\
& $\mathrm{He}$ & $5.82 \times 10^{-18}$ & $1.32 \times 10^{-16}$ & $4.81 \times 10^{-16}$ \\
\hline
\end{tabular}

* Rates for higher DF-levels scale as $\mathrm{v}^{1.9}$ for both helium and argon.

To arrive at a quantitative comparison, we have used a computer model to calculate laser performance with argon and helium as buffers. Our model contains approximately 200 reactions and over 40 different species or vibrational states. The kinetics of F-atom production are treated phenomenologically: we input a source term that describes the energy deposited per cubic centimeter per second into the gas, and we assume that one F-atom was produced for every $5 \mathrm{eV}$ deposited in the gas, regardless of whether the buffer was helium or argon (Sec. IV explores this issue). Our results showed that with the use of helium, the laser specific energy increased by $17 \%$ and pulse length shortened by about $0.7 \%$.

A reactor-driven laser, however, will use ${ }^{3} \mathrm{He}$ and not ${ }^{4} \mathrm{He}$, as discussed above. Because both species have nearly identical electronic structures, the isotopic subatitution should have little effect on the croas sections for kinetic processes. In fact, measured cross sections for deactivating $\mathrm{CO}_{2}(001)$ by ${ }^{3} \mathrm{He}$ and ${ }^{4} \mathrm{He}$ do exist and are identical. 11 Bimolecular kinetic rates, however, depend on a cross section and a collision frequency. The latter varies inversely with the square root of the reduced mass. For ${ }^{3} \mathrm{He}$ interacting with $\mathrm{CO}_{2}$ or DF, there is a net increase in the rate by $13 \%$ or $14 \%$, respectively. The scaling of three body rates is somewhat ambiguous and depends on the model chocen. $A$ simple-minded approach leads to increases in the rates for Reactions (4) and (5) of only 7\%.

Incorporating the above changes in kinetic rates to account for ${ }^{3} \mathrm{He}$, we repeated our previous calculation. The increases in the rate constants decreased specific power by $2.5 \%$ and shortened pulse length by $0.5 \%$. The net effect is that the DF- $\mathrm{CO}_{2}$ laser using ${ }^{3} \mathrm{He}$ should still out-perform an argon-based system.

By-products of the nuclear reaction may have another effect on the kinetics. The reaction: $\mathrm{n}+{ }^{3} \mathrm{He} \rightarrow{ }^{1} \mathrm{H}^{+}+{ }^{3} \mathrm{He}^{+}+2$ yields a proton and a triton at energies of $0.57 \mathrm{MeV}$ and $0.19 \mathrm{MeV}$, respectively. Assuming both these species eventually pick up an electron, we have the possibility of reactions with $\mathrm{F}_{2}$ to produce $\mathrm{HF}$ and $\mathrm{TF}$ in various vibrational states. Both $\mathrm{HF}-\mathrm{CO}_{2}$ and TF-CO2 have lased but have not performed as well as $\mathrm{DF}-\mathrm{CO}_{2}$ lasers. ${ }^{12,13}$ If we assume that the nuclear reaction deposits a total of $3 \mathrm{~mJ} / \mathrm{cm}^{3}$ in the gas, we can readily calculate from the numbers given above that the densities of 'TF and HF will be on the order of $1010 / \mathrm{cm}^{3}$. Because this is significantly below the level of DF densities encountered, both species will have little effect on laser performance. 


\section{EFFECT OF REACTOR PULSE SHAPE ON LASER PERFORMANCE}

Historically, DF-CO $\mathrm{CO}_{2}$ lasers have been initiated with flashlamps or an e-beam operating for, at most, a few microseconds. This is much shorter than the neutron pulse from the Los Alamos burst reactors: GODIVA (FWHM of $30 \mu \mathrm{s}$ ) and SKUA (FWHM of up to $400 \mu \mathrm{s}$ ). If the energy deposited (i.e., the total number of F-atoms produced) is kept constant but the initiation pulse is lengthened, fewer $F$-atoms are present at any one time to drive the chemistry. In this section we will examine the effect of initiation pulse length on laser performance.

We have estimated the total neutron fluence with the Los Alamos Monte Carlo code MCNP, ${ }^{14}$ based on a total of $5 \times 10^{16}$ fissions for GODIVA and $3 \times 10^{17}$ fissions for SKUA. Some aspects of the design were adjusted to attempt to make the energy deposition uniform throughout the laser. With 61 torr of ${ }^{3} \mathrm{He}$ in the laser, the neutrons can deposit about $3 \mathrm{~mJ} / \mathrm{cm}^{3}$ in the gas. As before, we assumed that one F-atom was produced for every $5 \mathrm{eV}$ deposited in the gas. The gas pressure was 603 torr, with the remaining gasses as given in Table II.

Our results are summarized in Figs. 1 and 2. Specific energy has been normalized for simplicity. We note that short initiation pulses ( $30 \mu \mathrm{s}$ FWHM) yield higher specific energies and shorter lasing pulses. As the initiation pulse broadens, the laser energy drops, and its pulse length becomes very long. (Laser pulse length as described in this document is measured from the onset of lasing to termination. Generally it is much longer than the FWHM of the lasing pulse.) A close examination of the shape of the laser output flux reveals a long gradual onset followed by a dramatic rise and abrupt termination. With very long initiation pulses, the lasing terminates at about the time that the neutron flux peaks.

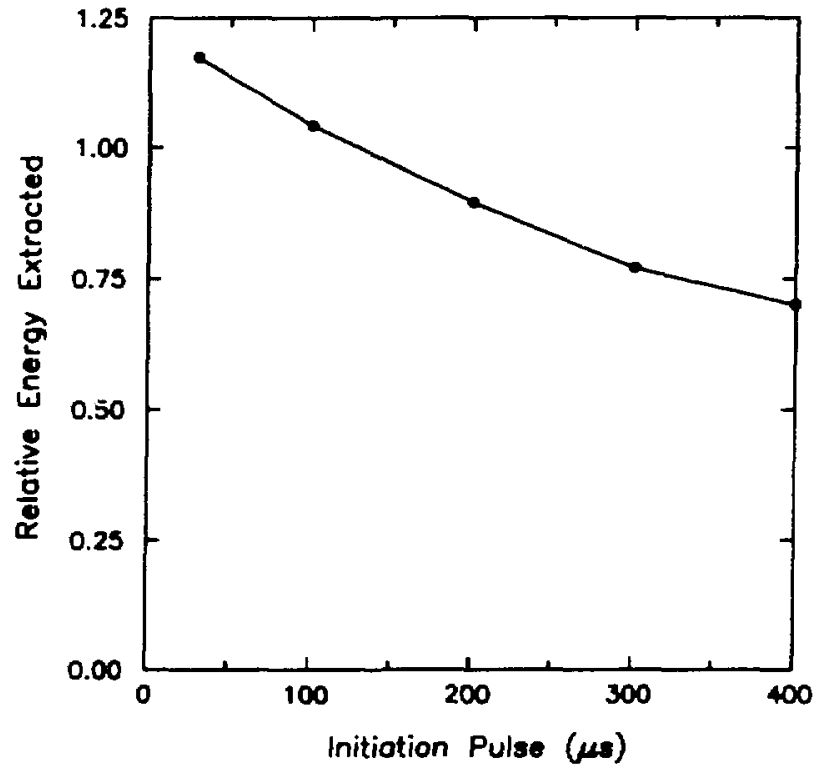

Fig. 1. Efficiency of energy extraction as a function of initiation pulse length. Total energy deposited was the same for all calculations.

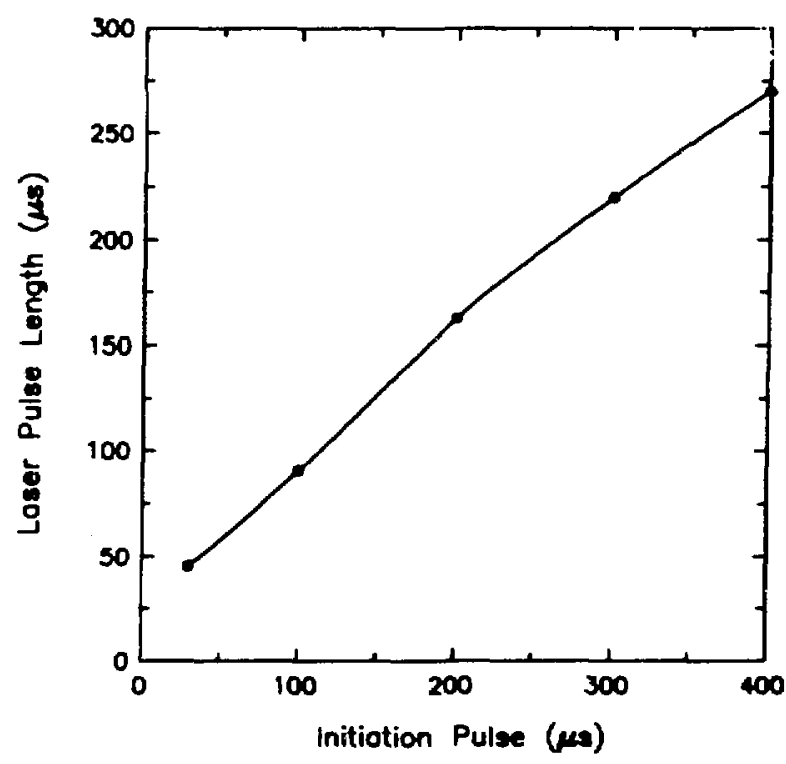

Fig. 2. Laser pulse length as a function of initiation pulse length. 
Increasing the concentration of ${ }^{3} \mathrm{He}$ in these calculations has the effect of increasing energy deposition and more strongly initiating the laser. We have repeated our calculations for the $400-\mu s$ initiation pulse with 332 torr of ${ }^{3} \mathrm{He}$ instead of the 61 torr considered previously. Though this raises the total energy deposition by a factor of 5.4, it yields only a $28 \%$ increase in specific energy and a $56 \%$ decrease in pulse length. This effect is much less pronounced than might be expected. Despite the large total energy deposition, the pulse length is so long that few F-atoms are available at any one time to drive the chemistry.

\section{EFFECT OF HELIUM ON F-ATOM PRODUCTION}

Dissociation of $F_{2}$ to produce free fluorine atoms and to initiate the chemistry of the transfer system can be accomplished either by UV-photolysis or an e-beam. The photolysis processes are straightforward: $F_{2}$ absorbs a photon and is excited to a repulsive state. The e-beam initiation is considerably more complex: high-energy electrons deposit their energy in the rare gas and produce metastable atoms, ions, and free electrons. A series of reactions of these species with $F_{2}$ generates the free F-atoms. The efficiency of this process is measured by the number of electron volts that must be deposited in the medium to create an F-atom. In previous sections of this report we have assumed a nominal value of $5 \mathrm{eV} / \mathrm{F}$-atom. If this number is significantly higher for the reactor-based system, higher neutron fluxes will be required to generate the same level of initiation.

The basic difference between the e-beam and reactor-pumped lasers is the replacement of argon by ${ }^{3} \mathrm{He}$. To better understand how this affects $\mathrm{F}$-atom production, we need to discuss e-beam initiation in more detail. In the following we will consider a laser volume containing mainly the rare gas and small amounts of $\mathbf{F}_{2}$.

As mentioned above, the high-energy electrons create argon ions, argon metastables, and secondary electrons. Low-energy electrons can very rapidly attach themselves to $F_{2}$ and produce a free $F$ and a free F. Argon ions attach themselves to the $F^{-}$to produce ArF*. This excimer is also produced by the reaction of $\mathrm{F}_{2}$ with argon metastables. The $\mathrm{ArF}^{*}$ will eventually react with $\mathrm{F}_{2}$ and produce three free F-atoms. Approximately $80 \%$ of the frce $\mathrm{F}$ produced in the Ar-F2 mixture comes from the reaction of $A r F^{*}$ and $F_{2}$. In a $\mathrm{He}-F_{2}$ mixture things are very different: the excimer $H e F^{*}$ is not bound, and this major channel in producing $F$-atoms will be cut off.

In He-F mixtures the major channel to $\mathrm{F}$-atom production comes through $\mathrm{F}_{2}{ }^{+}$. This species can be created either by charge exchange with helium ions or by Penning ionization with helium metastables. The $\mathrm{F}_{2}{ }^{+}$then combines with $\mathrm{F}^{-}$to produce three $\mathrm{F}$-atoms. In mixtures containing $\mathrm{He}_{2} \mathrm{~F}_{2}$, and $\mathrm{Ar}$, all the processes described above take place with charge exchange between helium ions and argon, as well as Penning ionization of argon by helium metastables.

We have used the model of Wilson and Shapiro ${ }^{15}$ to make quantitative comparisons of F-atom production in $\mathrm{He}, \mathrm{Ar}$, and $\mathrm{F}_{2}$ mixtures. This model incorporates a total of 18 species and 45 reactions. By making the major assumption that all electrons are instantly thermalized, we eliminate the need to include a Boltzmann analysis. Source terms that drive the kinetics come from the reaction of neutrons with ${ }^{3} \mathrm{He}$.

For the reactor-initiated laser, we want to compare $\mathbf{F}$-atom production in various $\mathrm{He}, \mathbf{A r}$, and $\mathrm{F}_{2}$ mixtures, with F-atom production in the e-beam-initiated Ar and $F_{2}$ mixture. By slightly modifying the Wilson and Shapiro model to treat an e-beam-driven $A r$ and $F_{2}$ mixture, we can calculate a base-line number for F-atom production in argon. This analysis yields a value of $5.2 \mathrm{eV} / \mathrm{F}$-atom, which is slightly higher than the approximate value of $5 \mathrm{eV} / \mathrm{F}$-atom used earlier. Losses of ArF* through radiation and collisional dissociation with argon sccount for much of the difference. 
We used the 5.2-eV/F-atom value to normalize our results for argon and helium mixes. Figure 3 reports the relative initiation strength needed for the same F-atom density in various mixtures. The total buffer gas pressure ( $\mathrm{He}+\mathrm{Ar}$ ) was fixed at 715 torr for these calculations. Though more energy deposition is required in pure helium than in pure argon, a few hundred torr of argon makes the F-atom production close to what it would be if only argon were present. Our calculations ignore a number of small contributions, and we have dropped the F-atom recombination process ${ }^{15}$ that artificially inflates the eV/F-atom number. In an actual laser, the F-atoms will be removed by reaction with $\mathrm{D}_{2}$ much more quickly than they can recombine.

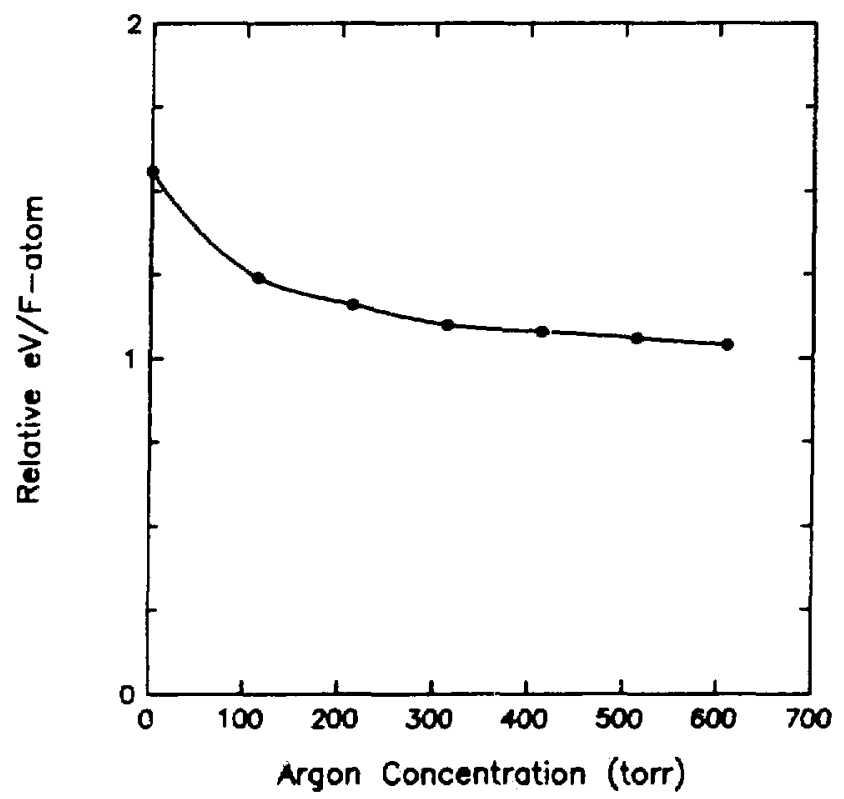

Fig. 3. Relative amounts of energy needed to produce one F-atom as a function of argon concentration. The total pressure is 715 torr, and the gas is composed of helium and argon. The results are normalized to one $(-5 \mathrm{eV} / \mathrm{F}$-atom) for a pure argon system.

Burrows ${ }^{16}$ has pointed out the additional processes that can occur when $\mathrm{D}_{2}$ and $\mathrm{CO}_{2}$ are included in Ar- $\mathrm{F}_{2}$ mixtures. The presence of helium metastables and ions further complicates the kinetics. Of major importance is the very rapid $\left(k \sim 10^{-9}\right)$ reaction between helium ions and $\mathrm{CO}_{2}$ to produce $\mathrm{O}^{+}, \mathrm{CO}$, $\mathrm{CO}^{+}$, and $\mathrm{O}^{15}$ The reaction between helium ions and $\mathrm{D}_{2}$ is slow and can be ignored. ${ }^{17}$ Reaction rates for helium metastables with $\mathrm{CO}_{2}$ and $\mathrm{D}_{2}$ have been measured. ${ }^{18}$ In the case of $\mathrm{D}_{2}$, the products are also known (mainly $\mathrm{D}_{2}^{+}$). ${ }^{19}$ Because both helium ions and metastables are very effective in producing $\mathrm{F}_{2}{ }^{+}$, these additional processes lower the production of $\mathrm{F}_{2}^{+}$and create instead $\mathrm{D}_{2}^{+}, \mathrm{CO}_{2}^{+}, \mathrm{O}^{+}$, and $\mathrm{CO}^{+}$. The most likely fate of these ions is reaction with the excess $F$ that will be present. Unfortunately, there are no measured rates for processes such as these.

In view of the uncertainties, we can make no prediction for the efficiency of F-atom production in actual laser mixtures ( $\mathrm{Ar}, \mathrm{He}, \mathrm{F}_{2}, \mathrm{D}_{2}$, and $\mathrm{CO}_{2}$ ). Our earlier analysis (Figs. 1 and 2) was based on 61 torr of ${ }^{3} \mathrm{He}$ in the gas, an energy deposition of $3.0 \mathrm{~mJ} / \mathrm{cm}^{3}$, and an assumed value of one $F$-atom produced for every $5 \mathrm{eV}$ deposited in the gas. If, however, it were to take $10 \mathrm{eV}$ to produce one F-atom, we need only increase the ${ }^{3} \mathrm{He}$ concentration to achieve the same level of initiation from the same reactor flux. This indicates that we have some leeway in achieving a specific level of laser performance, even if we do not know the level of initiation. Because laser performance is very 
sensitive to the level of initiation (among other things), the impact will be in our ability to make a reliable prediction.

\section{BURST REACTOR OPERATION}

Initiation pulses for the DF-CO $\mathrm{CO}_{2}$ laser are provided by one of two Los Alamos burst reactor facilities: GODIVA ${ }^{20}$ or SKUA. ${ }^{21}$

Figure 4 shows the GODIVA assembly and controls. The fuel is a 1.5-wt \% molybdenum-uranium alloy with $-93.5 \%$ enriched uranium. The fuel components are all aluminum-ion-plated and have a total mass of about $66 \mathrm{~kg}$. Three external C-shaped clamps (0.75 in. thick by $1.75 \mathrm{in}$. in cross section), fabricated from high-strength maraging steel, fasten the stack of six stationary fuel rings.

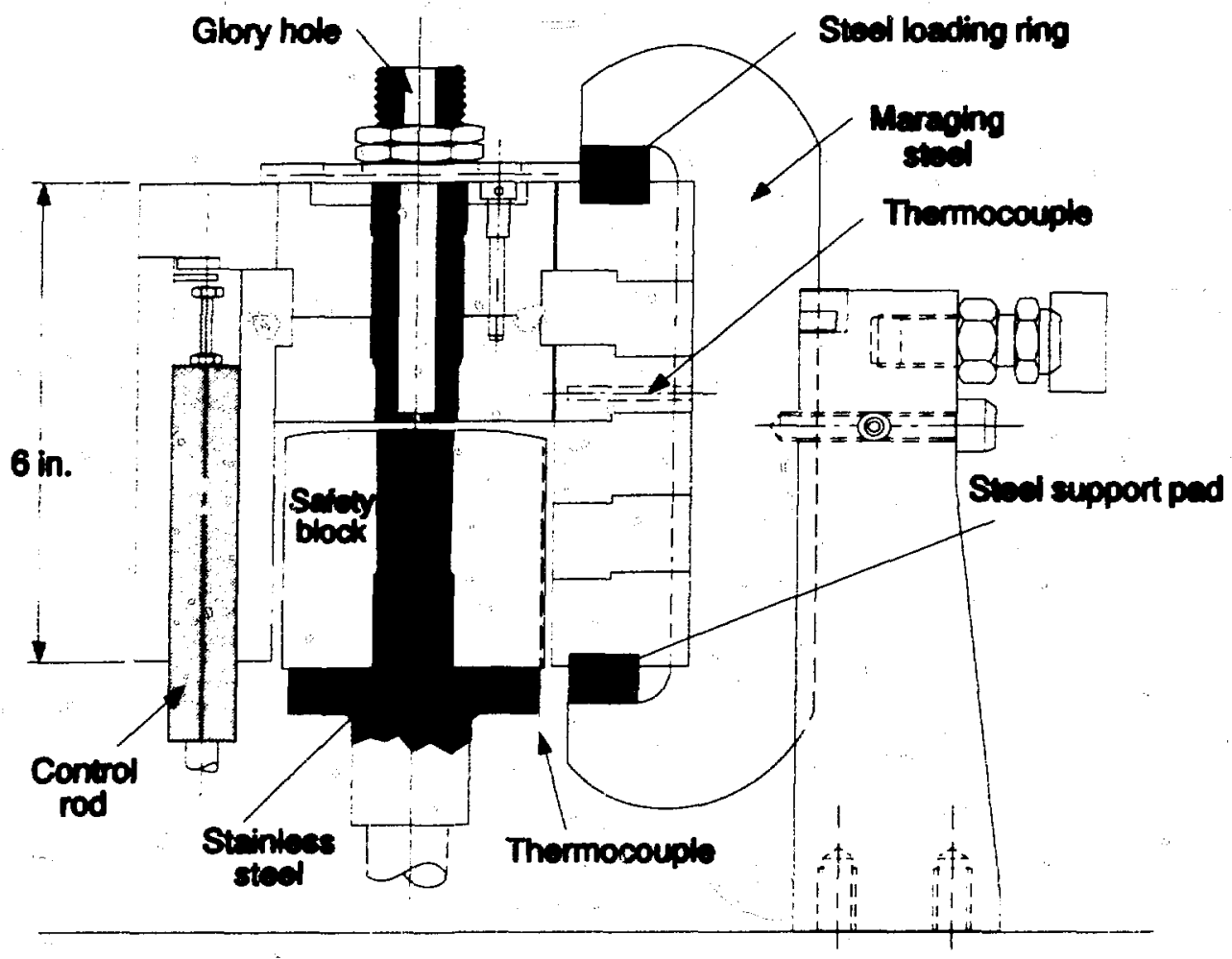

Fig. 4. GODIVA assembly and controls.

The burst assembly is supported on a three-legged structure that houses actuators for reactivity control elements, which enter the core from below. The safety block is threaded onto a stainless steel mandrel at the lower end, so that thermal expansion exerts a downward thrust on the support shaft, opening a magnetic clutch to provide shock-induced scramming. Total burst yield and burst width are plotted against reactor period in Fig. 5. Yield data and leakage flux per core fission have been obtained via foil activation and from aluminum $(n, \alpha)$ detectors. 


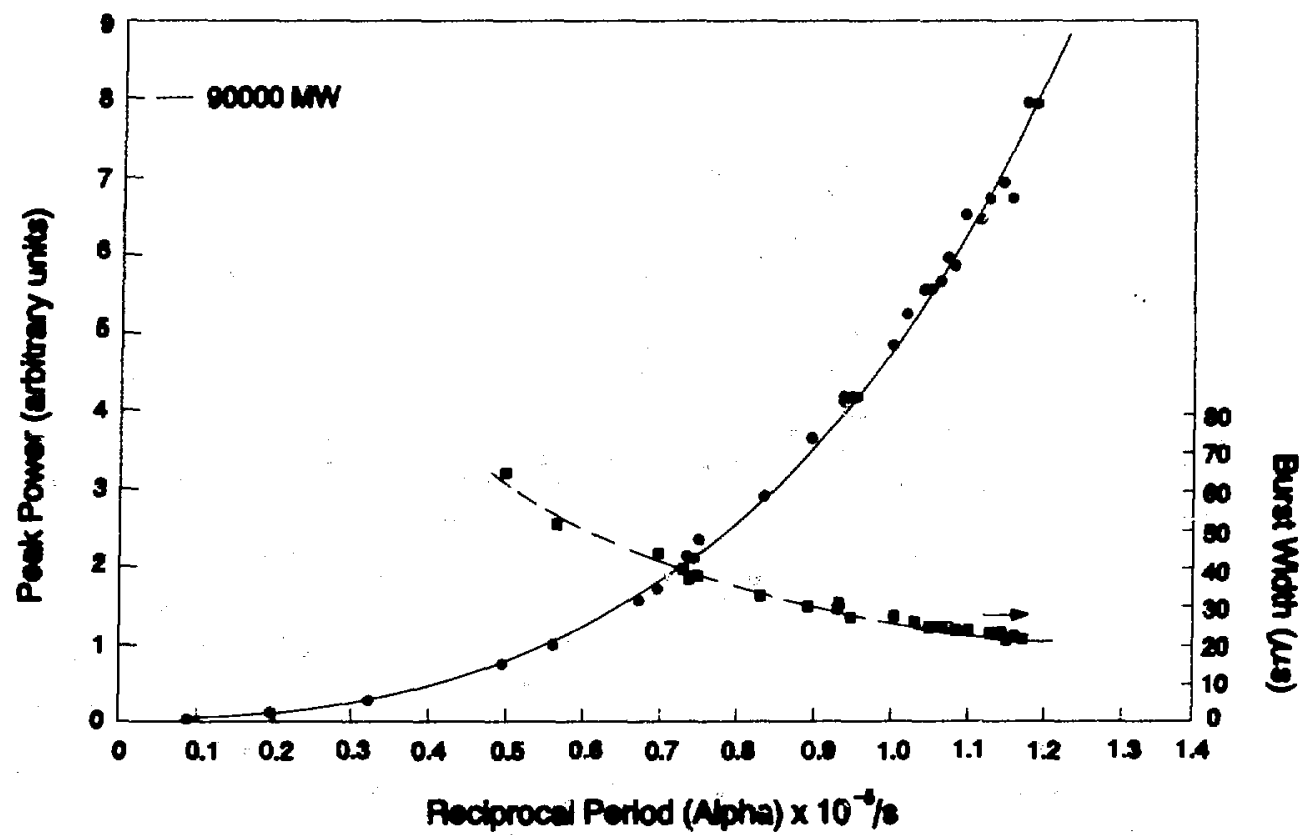

Fig. 5. Burst width and peak power of the GODIVA assembly plotted as a function of the reciprocal period. The dashed line is simply a guide for the eye. The solid curve has the functional form $\mathrm{P}=\left(\alpha^{2} / 2 b \alpha_{r}\right)\left(1+\alpha^{2} \tau^{2}\right)$, where $\mathrm{b}$ is the thermal quench rate of the device (about $-0.2 \mathrm{e}^{\circ} \mathrm{C}$ ), $\alpha_{\mathrm{r}}$ is the Rossi alpha (about $0.86 \times 10^{6} \mathrm{sec}^{-1}$ ), and $\tau$ has been adjusted for best fit to a value of $8.9 \mu \mathrm{s}$.

Figure 6 shows a top view of the SKUA assembly. This 18 -in.-diam cylindrical annulus, consisting of $170 \mathrm{~kg}$ of $93.5 \%$ enriched uranium, is controlled by six external copper reflector elements. A 9.5-in.-diam glory hole is available for experimental use. When a lower average-energy neutron spectrum is desired, a 3-in. annular flux trap with a 3-in. glory hole may be inserted. Though the SKUA assembly has not yet operated in the prompt critical regime, initial delayed critical operations have confirmed the SKUA design.

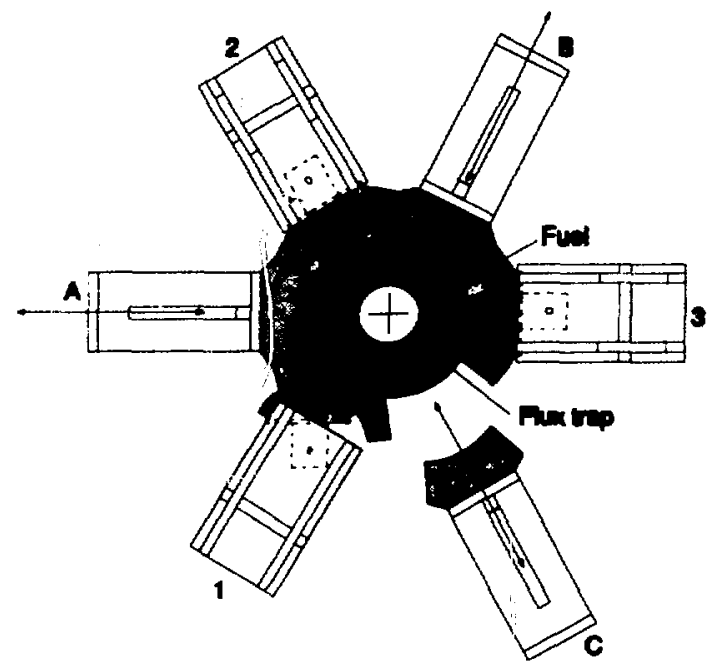

Fig. 6. Top view of the SKUA burst assembly. 
No special cooling following bursts is envisioned for either assembly, even though temperatures as high as $300^{\circ} \mathrm{C}$ (for GODIVA) or $500^{\circ} \mathrm{C}$ (for SKUA) may be achieved. The cool-down time for these devices defines the burst repetition rates that can be achieved.

A wide variation in irradiation pulse characteristics is possible using these facilities. Fission yields of between $1 \times 10^{16}$ and $3 \times 10^{17}$, with burst widths between 30 and $400 \mu \mathrm{s}$, are possible. Some of the burst properties of these two assemblies are summarized in Table IV; a typical short-burst pulse from the GODIVA assembly is shown in Fig. 7.

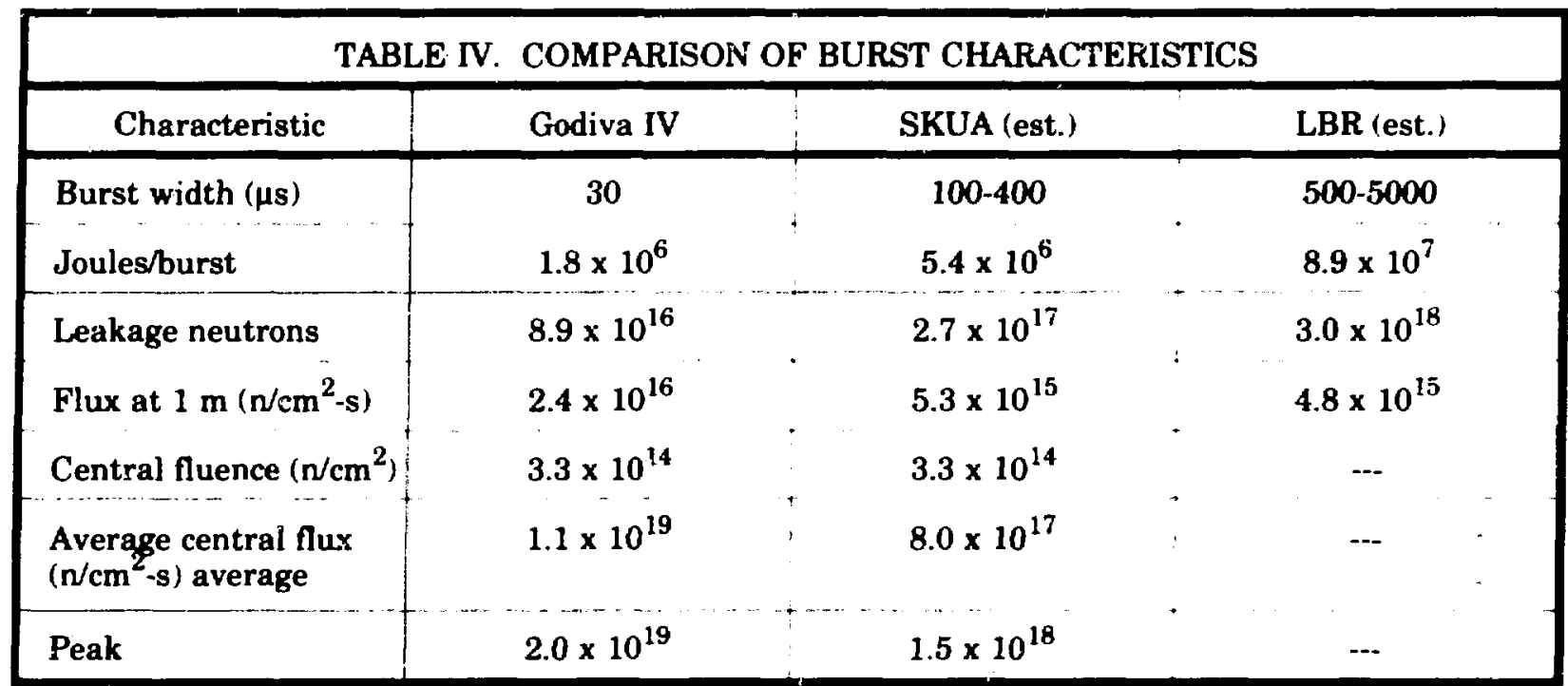

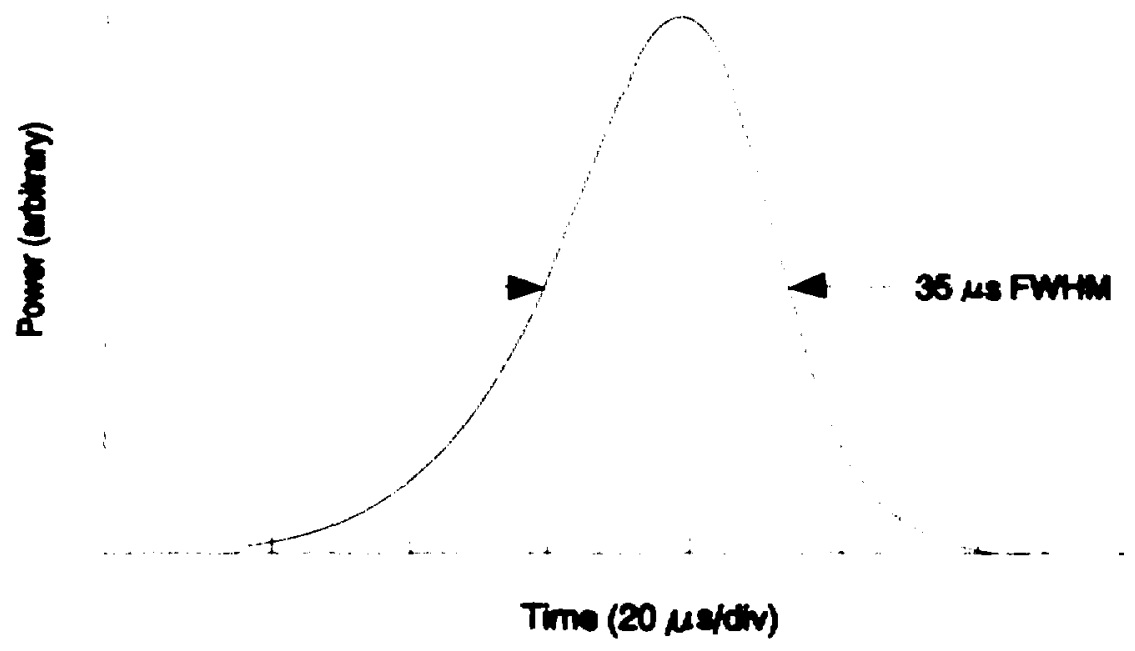

Fig. 7. A typical fast initiation pulse from the GODIVA assembly. 
Because these assemblies produce primarily fast neutrons, a moderator is necessary to thermalize the neutrons that enter the laser volume. The assemblies are relatively small, and the laser can be placed quite close to the source, which increases the efficiency of the system. On the other hand, a large laser volume will not be uniformly pumped under these circumstances. Some regularization of the neutron flux profile may be achieved by proper shaping of the moderator; however, this procedure produces some elongation of the initiation pulse length.

Energy deposition within the laser volume was estimated using the Los Alamos Monte Carlo code MCNP. ${ }^{14}$ Estimates were made for both the GODIVA and SKUA assemblies for several different laser volumes. Continuous-energy cross-section sets were used in the calculations, and the results were collapsed into 16 to 50 groups for analysis. A typical geometric model used in the calculations is shown in Fig. 8. Average energy depositions for several replacements of the argon buffer gas are shown in Table $V$. According to these results, minimum requirements for the ignition energy were met, with considerable room left for variation of the exact fill gas composition. However, we still must show that any degradation of the laser kinetics remains at an acceptable level. Degradation could occur from the alteration of the fill-gas composition, nonuniformities in the pumping profile, or longer initiation pulses provided by the burst assembly.

\begin{tabular}{|c|c|}
\hline \multicolumn{2}{|c|}{$\begin{array}{l}\text { TABLE V. ENERGY DEPOSITIONS ACHIEVABLE IN A } \\
\text { LARGE-SCALE DF-CO }{ }_{2} \text { LASER }\end{array}$} \\
\hline Additive & Energy Deposition \\
\hline none & $0.1 \mathrm{~mJ} / \mathrm{cm}^{3}$ \\
\hline 0.5 -atm ${ }^{3} \mathrm{He}$ & $4.5 \mathrm{~mJ} / \mathrm{cm}^{3}$ \\
\hline 0.1 -atm ${ }^{3} \mathrm{He}$ & $1.5 \mathrm{mel} / \mathrm{cm}^{3}$ \\
\hline 0.1 -atm ${ }^{10} \mathrm{~B}$ & $4.5 \mathrm{~mJ} / \mathrm{cm}^{3}$ \\
\hline $0.1-\operatorname{atm}{ }^{6} \mathrm{Li}$ & $3.5 \mathrm{~mJ} / \mathrm{cm}^{3}$ \\
\hline $0.1-\operatorname{atm}{ }^{235} \mathrm{U}$ & $50 . \mathrm{cmJ} / \mathrm{cm}^{3}$ \\
\hline
\end{tabular}

One of the objectives of the experimental program will be to study the characteristics of alternative types of burst systems that may be more suitable for use with a large-scale laser. Among the characteristics of such a system : vould be increased neutron output, increased burst repetition rate, and greater uniformity in the energy deposition within the laser volume. The size of the GODIVA and SKUA pulses is fundamentally limited by the mechanical stresses produced by thermal expansion of the fuel during the burst and, to a lesser extent, by the temperature rise in the fuel produced by the fission fragments. The pulse length is determined fundamentally by the physical size of the assembly.

A new liquid burst facility, the SHEBA assembly, is presently being constructed to study the dynamics of nuclear excursions in a liquid system. Compared to a metal system, the SHEBA assembly might ultimately produce an increase by a factor of 10 to 100 in the neutron output and a substantially higher burst repetition rate at the cost of a somewhat longer pulse length. In its final form, this liquid burst facility would have a substantial volume, and its shape would be tajlored to the requirements of the laser system. A critical mass of liquid material would be pumped into a shape of interest, then allowed to burst and vaporize. The material would then be cooled, reconstituted, and 
pumped back into the critical configuration for another burst. A comparison of the characteristics of such a liquid burst reactor (LBR) with GODIVA and SKUA has been included in Table IV.

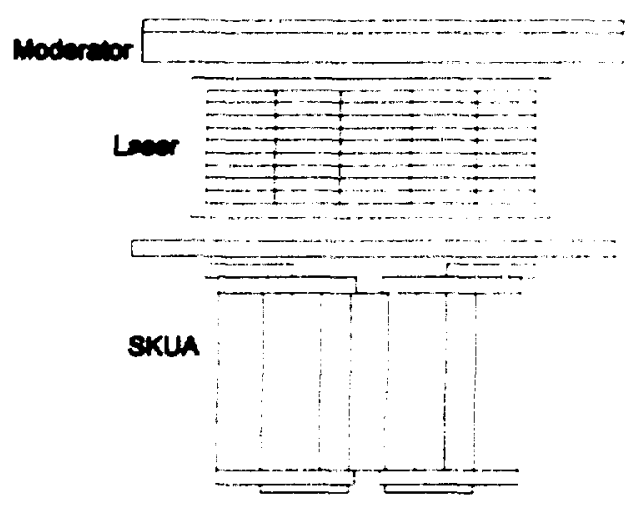

Fig. 8. Geometric model for determining the energy deposition within the laser during a typical burst from SKUA. The laser has been segmented to study the space and time profiles of the initiation pulse.

\section{PROPOSED EXPERIMINTTS}

A large-volume laser experiment is currently being planned to examine the issues of laser and burst assembly operation raised here. We propose to do proof-of-principle experiments whose objectives are

1. to demonstrate lasing in a neutron-initiated $\mathrm{DF}-\mathrm{CO}_{2}$ laser;

2. to examine the kinetics of the laser when various fractions of the buffer gas are replaced by ${ }^{3} \mathrm{He}$ and other gases;

3. to examine the feasibility of long pulse initiation (30- to $500-\mu \mathrm{s}$ range);

4. to produce a laser large enough to demonstrate optics performance and beam quality; and

5. to study the characteristics of solution burst assemblies.

We envision the use of two laser volumes. The smaller of the two will be approximately $40 \mathrm{~cm}$ in diameter and $2 \mathrm{~m}$ long. Using -20 -cm-diam optics, we will measure gain, total output energy, and pulse shape to verify and adjust performance predictions. Full-size, $40-\mathrm{cm}$-diam optics would then be used to show pumping uniformity and extraction over the full volume of $-280 l$.

A second laser would have a larger volume $\left(-2 \mathrm{~m}^{3}\right)$ with a folded optical path; an unstable resonator would extract the full volume. Preliminary calculations indicate that this volume will be large enough to give an equivalent Fresnel number well above the critical Fresnel number for mode separation, allowing the laser to produce a single plane wave. 


\section{CONCLUSIONS}

In this report, we have examined some of the issues that arise in attempting to initiate a large-volume, DF-CO $\mathrm{CO}_{2}$ transier laser with a burst reactor. While several issues remain to be settled in both the operation of the burst reactor and in the laser kinetics, analysis of a system in which ${ }^{3} \mathrm{He}$ is used to replace some fraction of the buffer gas indicates that excellent performance of the system may be expected.

\section{REFERENCES}

1. J. A. DeShong, "Optimum Design of High-Pressure, Large-Diameter, Direct Nuclear-Pumped Gas Lasers," Argonne National Laboratory report ANL-7030 (June 1965).

2. N. W. Jalufka, "Direct Nuclear-Pumped Lasers," NASA Technical Paper 2091 (January 1983).

3. M. A. Prelas, et al., "Nuclear Driven Flashlamps," Lasers and Particle Beams, 6, Part 1, 25-62 (1988).

4. G. York, Los Alamos National Laboratory, private communication (August 1988).

5. R. C. Feber and J. H. Sullivan, Los Alamos National Laboratory report LA-11049-MS (August 1987).

6. N. Cohen and J. F. Bott, Aerospace Corporation report SD-TR-82-86 (The Aerospace Corporation, EI Segundo, California, October 1982).

7. G. K. Vasil'ev, E. F. Makarov, and Yu, A. Chernyshev, Kin. i Kat. 16, 272 (1975).

8. A. S. Bashkin, A. N. Oreavskii, V. N. Tomashov, and N. N. Yuryshev, Sov. J. Quantum Electron. 10, $781(1980)$.

9. S. T. Amimoto, J. S. Whittier, G. Harper, R. Hofland, J. M. Wlaters, T. A. Barr, R. L. Kerber, and W. K. Jaul, Aerospace Corporation report SD-TR-83-05 (The Aerospace Corporation, El Segundo, California, February 1983).

10. H. Inagaki, K Kumamoto, A. Suda, M. Obara, and T. Fujioka, Inst. Phys. Conf. Ser. No. 72 , presented at 5th GCL Symp., (Oxford, England, 1984).

11. J. F. Stephenson, R. E. Wood, and C. B. Moore, J. Chem. Phys. 54, 3097 (1971).

12. V. Y. Argoskin, G. K. Vasil'ev, V. I. Kir'yanov, and V. L. Tal'roze, Sov. J. Quantum Electron. 8, 1366 (1978).

13. A. I. Nikitin and A. N. Oreavskii, Sov. J. Quantum Electron., 6, 1280 (1976).

14. "MCNP - A General Monte Carlo Code for Neutron and Photon Transport," J. Briesmeister, Ed., Los Alamos National Laboratory report LA-7396-MS, Rev. 2 (September 1986).

15. J. W. Wilson and A. Shapiro, J. Appl. Phys. 51, 2387 (1980). 
16. M. D. Burrows, Los Alamos National Laboratory, private communication (December 1987 ).

17. F. C. Fehsenfeld, A. L. Schmeltekopf, P. D. Goldan, H. I. Schiff, and E. E. Ferguson, J. Chem. Phys. 44, 4087 (1966).

18. A. L. Schmeltekopf and F. C. Fehsenfeld, J. Chem. Phys. 53, 3173 (1970).

19. L. T. Specht, K. D. Foster, and E. E. Muschlitz, J. Chem. Phys. 63, 1582 (1975).

20. T. F. Wimett, "Fast Pulse Reactor Kinetics: Theory and Experiment," Proceedings of the US/Japan Seminar on Fast Pulse Reactors, January 1976 (Nuclear Engineering Research Laboratory, University of Tokyo, Tokai).

21. J. D. Orndoff, H. C. Paxton, and T. F. Wimett, "Safety Analysis of the Los Alamos Critical Experiments Facility: Burst Operation of SKUA," Los Alamos Scientific report LA-6206, Vol. II, Addendum (Dec. 1980). 